\title{
Neural representations of location outside the hippocampus
}

\author{
James J. Knierim
}

Department of Neurobiology \& Anatomy, W.M. Keck Center for the Neurobiology of Learning and Memory, University of Texas Medical School at Houston, Houston, Texas 77225, USA

\begin{abstract}
Place cells of the rat hippocampus are a dominant model system for understanding the role of the hippocampus in learning and memory at the level of single-unit and neural ensemble responses. A complete understanding of the information processing and computations performed by the hippocampus requires detailed knowledge about the properties of the representations that are present in hippocampal afferents and efferents in order to decipher the transformations that occur to these representations in the hippocampal circuitry. Neural recordings in behaving rats have revealed a number of brain areas that contain place-related firing properties in the parahippocampal regions and in other brain regions that are thought to interact with the hippocampus in certain behavioral tasks. Although investigators have just begun to scratch the surface in terms of understanding these properties, differences in the precise nature of the spatial firing between the hippocampus and these other regions promise to reveal important clues regarding the exact role of the hippocampus in learning and memory and the nature of its interactions with other brain systems to support adaptive behavior.
\end{abstract}

In the 1950s, a patient known as H.M. had much of his hippocampus and medial temporal lobe surgically removed in order to relieve the focus of his severe epilepsy. An unexpected consequence of the surgery was that H.M. lost the ability to form new, conscious (declarative) memories. Since the original report of this case (Scoville and Milner 1957), the hippocampus has been at the forefront of research into the biological basis of learning and memory. Although most researchers agree that the hippocampus plays a crucial role in the normal formation and long-term storage (or transfer to long-term storage) of explicit memories, the exact nature of its role has been the subject of intense debate. In one view, the hippocampus is theorized to be essential for declarative memory (Squire 1987), that is, the conscious recall of specific experiences from one's past (episodic memory) or of general facts about the world (semantic memory). In nonhuman animals, the term "relational learning" was coined to describe the types of hippocampus-dependent memory processing that would lead to declarative memory in humans (Cohen and Eichenbaum 1993). In another view, the hippocampus is theorized to be the locus of a "cognitive map" of the organism's environment(s), required not only for spatial learning and navigation but also as an organizing framework to locate and interrelate the items and events of experience (O'Keefe and Nadel 1978). Support for the latter view derived in large part from the existence of place cells in the rat hippocampus (O'Keefe 1976). These cells were specifically and selectively active when the rat occupied restricted locations in its environment, and the ensemble activity of these neurons was thought to be the neural instantiation of Tolman's hypothesized cognitive map (Tolman 1948). Subsequently, similar properties were found in primate hippocampus, including humans (Nishijo et al. 1997; Rolls et al. 1997; Ekstrom et al. 2003; Ludvig et al. 2004).

The first two decades of research on place cells focused on (1) describing the types of sensory input that generated or controlled their spatial firing fields (place fields) and (2) determining whether these cells only encoded space or whether spatial rela-

E-mail james.j.knierim@uth.tmc.edu; fax (713) 500-0621.

Article is online at http://www.learnmem.org/cgi/doi/10.1101//m.224606. tionship was merely a good example of a more general type of processing performed by the hippocampus (O'Keefe 1999; Shapiro and Eichenbaum 1999). Studies in recent years indicate that a resolution to this debate may be emerging, as concepts such as episodic memory, context-dependent learning, learning of spatiotemporal sequences, etc., can be viewed as requiring an integration of explicitly spatial and nonspatial representations (Nadel et al. 1985; Levy 1996; Mehta et al. 1997; Wallenstein et al. 1998; Redish 1999; Huxter et al. 2003; Eichenbaum 2004; Lee et al. 2004; Jensen and Lisman 2005). Accumulating evidence suggests that the firing of CA1 place cells may reflect the encoding of behaviorally salient, nonspatial information onto the spatial framework provided by place cells (O'Keefe 1976; Wiebe and Staubli 1999; Wood et al. 1999; Huxter et al. 2003; Moita et al. 2003; Hargreaves et al. 2005; Leutgeb et al. 2005). Nonetheless, further advancement is limited by the relative paucity of knowledge of the properties of neurons that interact with the hippocampus. To understand the specific role of the hippocampus in learning and memory, it is critical to have an understanding of the neural representations already present in its afferent structures, how these representations are altered within the hippocampus and within its efferent structures, and how different brain systems interact with the representations of the hippocampus and medial temporal lobe. Slowly but steadily, a number of research groups have been filling this gap.

There are a number of possible ways in which hippocampal neural firing properties may reflect learning and memory processes. The most straightforward possibility is that hippocampal firing may change in direct correlation with performance of a particular learning task (e.g., classical conditioning) (Berger et al. 1976; McEchron and Disterhoft 1997; Moita et al. 2003). In other tasks, the hippocampal neurons may not change their firing properties in a way that is obviously related to the learning protocol, but they may instead provide unique representations that other brain areas require in order for the animal to learn the task at hand. For example, the hippocampus may create a unique contextual or configural representation that is used to bind together the various aspects of experience that are represented in extrahippocampal (e.g., neocortical) areas (O'Keefe and Nadel 1978; Teyler and DiScenna 1985; Sutherland and Rudy 1989; 
McClelland et al. 1995). This unique representation may be a critical input that allows the extrahippocampal areas to change their neural firing properties in adaptive ways that directly modify the animal's behavior or to integrate neural firing changes across multiple brain regions, even though the hippocampal representation itself may not change after the initial formation and consolidation of its representation (Frank et al. 2004) In order to understand the function of the hippocampus in learning and memory-whether this function manifests itself in neural changes correlated with learning or in unique representations that are prerequisites for neural plasticity in other regions-it is imperative to determine whether the properties of hippocampal representations arise from hippocampal processing per se or whether they are already present in the hippocampal inputs. Because the spatially selective firing of place cells is the most striking correlate of hippocampal cells in the rat, it makes sense to begin this process by understanding the types of spatial information already present in hippocampal afferents, to understand what information is added by hippocampal computations, and to understand what properties are present in hippocampal efferent structures, reflecting the transformations that occur as the result of hippocampal processing. These data will allow the testing of computational models of hippocampal processing, which will hopefully lend experimental support and insight into theoretical constructs of hippocampal function that have existed for decades (e.g., Marr 1971; McNaughton and Morris 1987; Rolls and Treves 1998).

Accordingly, this review describes the spatial firing properties of neurons recorded from multiple brain regions outside the hippocampal formation in the rat (Fig. 1). Specifically, it concentrates on the properties of spatially tuned neurons in the direct afferent and efferent pathways of the hippocampus (the entorhinal cortex; the perirhinal and postrhinal cortex; the subiculum, parasubiculum, and presubiculum; and the septum) and in selected neural systems that interact with the hippocampal system (the prefrontal cortex, the striatum, the parietal cortex, and the superior colliculus). To maintain focus, the review does not cover studies that characterize correlates other than space, such as direction, behavior, sensory responses, etc., nor does it cover spe-

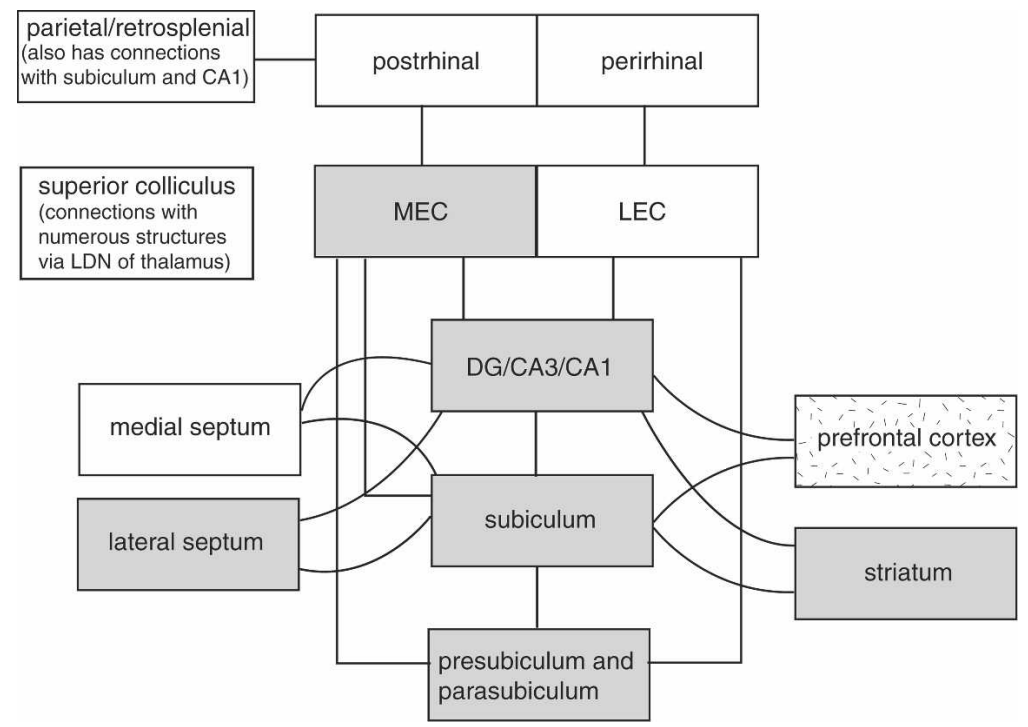

Figure 1. Simplified wiring diagram of the brain areas covered in this Review. Not all connections are diagrammed. Shaded regions represent brain areas that have been reported to contain place-related neural activity. Hatching of prefrontal cortex indicates that, although under restricted conditions place-related activity has been found, the cells seem to represent only goal-related locations (Hok et al. 2005). cies other than the rat. Hopefully, this review will serve as a clarion call for further research on these areas. Such research will be necessary to develop a more complete understanding of the exact nature of the computational processing provided by the hippocampus and the role of that processing within the larger framework of behavior that depends on multiple brain systems in the behaving animal.

An important caveat is that spatially correlated firing of neurons in behaving animals may arise from a number of sources that are not necessarily involved in spatial processing per se. For example, a neuron that encodes a particular behavior may fire preferentially in a particular location if the rat has a bias for performing that behavior in that location. Similarly, a cell that responds to a particular sensory cue may fire preferentially in a particular location if that cue is concentrated (or selectively available) at that location. Years of research on hippocampal place cells have disproved the notion that the spatially selective firing of these cells is an epiphenomenon or byproduct of some other, more explicit, response characteristic of these cells. For example, place cells show specific, spatially restricted firing fields under conditions in which behavior is homogeneous across an environment (Muller and Kubie 1987; Muller et al. 1987), and the firing fields are not tied to any particular, external sensory input (O'Keefe and Conway 1978; O'Keefe and Speakman 1987; Knierim et al. 1995). For most extrahippocampal areas, too few experiments have been performed to rule out the possibility that the spatially correlated firing in these areas arises as a secondary correlate from some other factor that correlates with a particular location (or set of locations). Thus, for some of the studies reviewed here, the spatially correlated firing must be tested further to determine whether the cells represent space in an allocentric framework, as do hippocampal cells, or merely reflect some other variable that is correlated with spatial location.

\section{Hippocampal afferent and efferent pathways}

\section{Medial entorhinal cortex}

The major source of cortical input to the hippocampus is the entorhinal cortex, which comprises two distinct regions-the medial entorhinal cortex (MEC) and the lateral entorhinal cortex (LEC) - that differ in their cytoarchitecture, connectivity, and neural responses (Witter and Amaral 2004). Early investigation of the MEC suggested that neurons in this area displayed modest spatial selectivity (Barnes et al. 1990; Mizumori et al. 1992). The most comprehensive early study was by Quirk et al. (1992), who investigated the firing of superficial MEC neurons while rats performed a pellet-chasing task in a high-walled chamber. This task is a standard protocol in the place field literature, allowing a direct comparison of entorhinal firing patterns with hippocampal place cells recorded under nearly identical conditions. Although Quirk et al. (1992) found that a minority of MEC neurons had place fields that were similar to CA1 place fields, on average, the MEC cells displayed less-precise spatial tuning than CA1 cells. Many neurons fired at high rates throughout the environment, but displayed a spatial bias for a particular location (similar to theta cells of the 
hippocampus). These firing patterns were stable within a session and across sessions, showing that the spatial signal was reliable. Like place cells, the orientation of the MEC firing field could be controlled by the rotation of salient landmarks in the periphery, but these landmarks were not necessary to support the spatially biased firing, which persisted even in the dark. As a result of these early studies, the view took hold that the major cortical input from the MEC represented spatial locations in a coarse fashion, with noisy cells exhibiting, on average, a modest spatial bias in their firing. The hippocampus presumably transformed this noisy input into the sharp, highly specific place fields that formed the hippocampal "cognitive map."

This notion was completely overturned with the recent discovery of the entorhinal "grid cell" (Fyhn et al. 2004; Hafting et al. 2005). In the most important finding regarding place cells in the past $20 \mathrm{yr}$, these researchers described a class of cells in the MEC that fire in an exquisitely precise, triangular grid pattern in an environment (Fig. 2). As a rat moves through an environment, the MEC neuron fires in multiple spots in that environment. Each spot is located at the vertex of a grid made of equilateral triangles that covers the entire floor of the environment. If the walls of the environment are removed and the rat is allowed to explore outside of the original boundaries, the grid is extended accordingly, presumably ad infinitum. Anatomically adjacent neurons display grids at similar spatial scales and orientations, reminiscent of the columnar structure of neocortex. However, the phase of the grid can be different between nearby cells (i.e., the grids of two cells show the same spacing and orientation, but one is shifted relative to the other). Thus, a small region of the MEC contains grid cells that cover the entire floor of the environment at a particular spatial scale and orientation. As the electrode moves to a new location, the orientation of the grid changes. Importantly, the scale of the grid changes systematically-as the electrode moves from the dorsocaudal MEC to more ventral MEC, the spacing of the grid becomes larger. The topography of projections from the MEC to the hippocampus follows this same gradient, as the dorsocaudal MEC projects to the most septal (dorsal) hippocampus and the ventral MEC projects to the most temporal (ventral) hippocampus (Dolorfo and Amaral 1998). Thus, high-resolution grid cells project to the dorsal hippocampus, where place fields are very specific, and lowresolution grid cells project to the ventral hippocampus, where place cells are less specific (Jung et al. 1994; Maurer et al. 2006).

This remarkable phenomenon has excited not only the hippocampus/place cell community, but systems neuroscientists in general. For place cell aficionados, the grid cell is a breakthrough finding that represents a third major type of cell that conveys fundamental information about space. The first cell type was the place cell, discovered in 1971 by O'Keefe and Dostrovsky (1971). The second type was the head-direction cell, discovered by Ranck Jr. in 1984, which serves as an "internal compass" to provide an orientation signal to the spatial representation (Ranck Jr. 1985; Taube et al. 1990a,b). The addition of the grid cell to this cast provides an enormously important piece of information critical for understanding the computations that the brain uses to create spatial representations. The crystalline structure of the grid-cell firing pattern seems unlike any other single-unit correlate observed in the brain, thus intriguing systems neuroscientists of all stripes. Although the grid can be aligned and oriented relative to external sensory cues, the grid structure itself is not dependent on any source of external sensory input (Hafting et al. 2005). That is, there is no external landmark (or configuration of landmarks) that could possibly drive these cells to fire in such a regularly repeating pattern. Rather, the grid cell is almost certainly a product of internally generated signals and computations, and probably is part of the brain system that computes the animal's location relative to a fixed reference point by means of path integration (Mittelstaedt and Mittelstaedt 1980; Gallistel 1990; Etienne 1992; McNaughton et al. 1996; Hafting et al. 2005; O'Keefe and Burgess 2005; Fuhs and Touretzky 2006). Each grid cell is presumably part of a network that keeps track of the rat's location at different spatial scales by integrating direction and speed-of-movement information to continuously update the rat's position relative to its starting point or to some other fixed reference location. The direction and speed representations can be derived from purely internal mechanisms, such as the vestibular system, motor efference copy, or other self-generated signals. Although the rat's location cannot be determined uniquely from the firing of a single grid cell, a population of grid cells can localize the rat with precision (Fyhn et al. 2004). This precision may arise from two factors. First, individual grid cells do not fire at the same rate at every vertex of the grid, but rather, show reproducible variation in firing rate across the vertices. Second, as mentioned above, different cells display grids at different spatial scales and orientations. The result of these two factors is that, at the neural ensemble level, each location can be represented by a unique vector of grid-cell firing rates, thereby allowing an accurate representation of the rat's current location. Although the neural circuits that produce the grid cell firing properties are not yet known, it is of inestimable importance that we now know, at a phenomenological level, the
Figure 2. Grid cells in the medial entorhinal cortex (Hafting et al. 2005). The triangular grid-firing structure of clusters of three neurons in the dorsocaudal MEC and in more ventral MEC are shown. The red cell of each cluster is diagrammed with dashed lines connecting some of the vertices to illustrate the equilateral triangular (or hexagonal) nature of the grid. Each grid cell in a cluster shares the same spacing and orientation, but the grids are displaced in phase. Grids at more ventral locations have a larger spacing between the vertices (i.e., a lower spatial resolution). High-resolution grid cells project to the dorsal hippocampus, while lower-resolution grid cells project to more ventral regions of the hippocampus.
Learning \& Memory www.learnmem.org 
nature of the firing patterns that provide a major input into the hippocampus, and this will allow us to understand more fully the nature of the processing that the hippocampus performs on this input.

\section{Do standard place cells exist in MEC?}

Hargreaves et al. (2005) recorded from the MEC in a region that probably did not project as far septally in the hippocampus as the region where Hafting et al. (2005) found the highest resolution grid cells, but certainly projected to a region within the dorsal part of the hippocampus. Like Quirk et al. (1992), these authors found a number of cells with specific, single-peaked place fields. Other cells had 3-4 peaks, and these peaks looked like they might be parts of a grid that represents locations at a scale about the size of the recording chamber. Importantly, Hargreaves et al. (2005) also recorded two bona-fide, high-resolution grid cells, thereby providing an independent replication of the phenomenon. Although they were not able to assign this recording site to the MEC with certainty, as it was in a region where the MEC, retrosplenial cortex, and parasubiculum meet, it is noteworthy that this recording came from the most caudally positioned electrode in their sample (i.e., in the region recorded by Hafting et al. 2005). One important question that remains is whether the single-peaked place fields recorded in MEC by Hargreaves et al. (2005) and by Quirk et al. (1992) were true place fields, similar to those of CA3 and CA1, or whether they were vertices of grids at such a low spatial resolution that the recording chamber enclosed only one vertex (or a fraction of a vertex). Since Hafting et al. (2005) did not report any classic place cells, it is important to know whether the MEC contains both grid cells and place cells, and whether the preponderance of these cells changes depending on the exact location of recording in the MEC.

\section{Lateral entorhinal cortex}

Some studies of spatial selectivity of the entorhinal cortex did not specify whether the recordings were from LEC or MEC (Barnes et al. 1990; Frank et al. 2000). One study (Mizumori et al. 1992) that showed slim spatial tuning of entorhinal neurons recorded from both LEC and MEC and reported that the firing properties of the two areas did not differ. The LEC follows a similar topography of projections to the hippocampus as the MEC (lateral LEC near the rhinal sulcus projects to dorsal hippocampus, while more medial LEC projects to ventral hippocampus) (Dolorfo and Amaral 1998), and these studies did not specify which projection band was recorded. Thus, it was an open question whether LEC neurons that projected to dorsal hippocampus contained spatially tuned neurons. Hargreaves et al. (2005) recorded from CA1, MEC, and LEC in corresponding projection bands that projected to dorsal hippocampus in a pellet-chasing task (Muller et al. 1987) similar to that of Hafting et al. (2005). As mentioned above, some MEC cells were apparently medium-resolution grid cells, while other cells had apparent well-formed, single-peaked place fields (like Quirk et al. 1992). In contrast, no LEC cells showed stable, highly specific place fields. Two cells showed spatially correlated firing, one at an edge of a salient cue card and the other in the center of the apparatus, where the chocolate reward pellets dropped. These two cells may be examples of spatially correlated firing that arises from other cues that happened to be correlated with a particular location. On average, the firing of LEC cells demonstrated much less spatial tuning than the firing of MEC or CA1 cells. Because LEC receives a major input from the perirhinal cortex (Burwell 2000; Witter et al. 2000), which appears to be part of the ventral, object-related (what) pathway, it is possible that LEC neurons convey information about particular objects in the environment (consistent with the cue-card and reward-site correlates), which the hippocampus combines with the spatial input from MEC to form conjunctive, object-in-place (or object-in-context) representations. This may be the rat analog of more general event + context or item + source representations that may underlie episodic memories in humans (Suzuki et al. 1997; Gaffan 1998; Davachi et al. 2003; Norman and Eacott 2005). Alternatively, it is possible that the landmark-poor environment of the recording chamber was not sufficient to drive spatially correlated firing in LEC. That is, LEC neurons may encode spatial locations by configurations of distal landmarks, and a single cue card may not provide enough information for these cells to fire selectively. In a more complex visual environment, or in an environment in which behavioral trajectories are more constrained, it is possible that LEC neurons might exhibit place fields similar to those of the hippocampus. Identifying the proper firing correlates of LEC neurons represents one of the next major challenges for understanding the inputs to the hippocampus and the computations performed by the hippocampus on these inputs.

\section{Path equivalence}

A major motivation for understanding the properties of entorhinal neurons is to decipher the computations performed by the hippocampus on its entorhinal input. Thus, one wants to understand the properties of hippocampal input, output, and internal representations and the rules that govern the transformations of these representations from one level of processing to another. In one such study (Frank et al. 2000), rats ran a variety of U- or $\mathrm{W}$-shaped tracks. Two interesting properties of both entorhinal and CA1 cells were reported. (1) Both sets of cells showed properties that Frank et al. (2000) termed "retrospective" or "prospective" coding, in that the firing rate of a cell as the rat ran through a particular place was modulated by whether the rat was about to make a particular turn (prospective coding) or whether the rat had just made a particular turn (retrospective coding). Although CA1, superficial EC, and deep EC all showed this effect, these response properties were more prevalent in EC than in CA1 (see also Wood et al. 2000; Ferbinteanu and Shapiro 2003) (but also Lenck-Santini et al. 2001; Bower et al. 2005). (2) Entorhinal cells, but not CA1 cells, showed a property that Frank et al. (2000) termed "path equivalence." That is, the entorhinal cells fired at multiple locations on a track or on each of two tracks, but each location corresponded to a region in which the trajectory of the animal was similar (e.g., when the animal was moving along the long stretch of the track and made a right turn). This property was present in both superficial and deep EC, suggesting that the EC may generalize similar trajectories across environments or locations in an environment, whereas CA1 maintains orthogonal representations of these trajectories, depending on the specific location or context. One critically important question is to understand how these properties, recorded on narrow tracks, relate to the grid-cell phenomenon (Fyhn et al. 2004; Hafting et al. 2005), recorded on two-dimensional platforms with lessconstrained behavioral trajectories. Further studies that demonstrate differences between the hippocampus and EC, especially those that find properties that are absent in the superficial (input) layers but present in the hippocampus and the deep (output) layers, will provide valuable information for deciphering the information processing that occurs in the hippocampus. Of course, recurrent connections and direct interactions between the deep and superficial layers of EC complicate this task (Kloosterman et al. 2003; Van Haeften et al. 2003), but characterizing the differences among these regions is a first step toward understanding the computations performed by the hippocampus and the neural circuitry that performs this processing.

\section{Perirhinal and postrhinal cortex}

The MEC and LEC are components of two relatively segregated processing streams that convey input to the hippocampus (Bur- 
well 2000; Witter et al. 2000). The MEC receives a major input from the postrhinal cortex (the rodent homolog of the parahippocampal cortex of primates) (Burwell et al. 1995), which is connected to the visuospatial areas of the parietal and retrosplenial cortex and appears to be part of the dorsal (where) pathway of primates. The LEC receives its primary input from the perirhinal cortex, which is connected to the object-related areas of the ventral (what) pathways of primates. Although there is significant cross-talk between these pathways, there is also a strong degree of anatomical segregation. Given the lack of spatial tuning of LEC neurons (Hargreaves et al. 2005), it is not surprising that perirhinal cortex neurons do not appear to demonstrate strong spatial selectivity as well. Some perirhinal neurons have been shown to fire on a +-maze or a T-maze in a spatially-biased manner, but in both cases, the firing patterns were not stable across sessions (Burwell et al. 1998; Zironi et al. 2001). Thus, it is unlikely that these cells carry a robust spatial signal, but rather they may be responsive to other factors that correlate with space in a single session. Furthermore, in a pellet-chasing task, perirhinal neurons show little, if any, spatial tuning, even within a session (Zironi et al. 2001; Hargreaves et al. 2005). Perirhinal neurons are more likely to respond to nonspatial stimuli, such as objects or auditory stimuli (Zhu et al. 1995; Suzuki et al. 1997; Wan et al. 1999; Lindquist et al. 2004).

Because the MEC contains grid cells, it is of great interest to determine whether postrhinal cortex also contains grid cells or some other spatial signal. Like perirhinal cortex, it appears that there is little, if any, consistent spatial firing of postrhinal neurons. On a +-maze, a greater proportion of postrhinal neurons showed a moderate amount of spatial tuning compared with perirhinal neurons, but the spatial correlates were not stable between recording sessions (Burwell and Hafeman 2003). Also, in a pellet-chasing task, there was little evidence of spatial tuning of postrhinal neurons (Fyhn et al. 2004). This result is somewhat surprising, given that the postrhinal cortex is thought to be part of a visuospatial processing stream (Burwell et al. 1995). Further experiments are necessary to determine the correlates of neural firing in this region. It is possible that changes in attention, eye movements, or other factors masked a more reliable spatial signal in the postrhinal cortex under the conditions in which these experiments were run. Nonetheless, it is clear that the MEC does not inherit its grid-cell firing properties from its postrhinal afferents.

\section{Presubiculum and parasubiculum}

The subicular complex comprises a number of areas, including the subiculum, the parasubiculum, and the presubiculum. The dorsal presubiculum is regarded by some as a distinct area, called the postsubiculum. The subiculum is the major recipient of CA1 axons, and is thus considered a primary output region of the hippocampus. Because the subiculum sends projections to the other areas of the subicular complex, these other areas have generally been considered to be hippocampal output targets as well. However, Witter and Amaral (2004) have argued, based on anatomical connectivity, that the parasubiculum, presubiculum, and postsubiculum should be regarded as hippocampal input areas. For example, the parasubiculum projects to layer 2 of both MEC and LEC, and layer 2 is the source of EC input to the dentate gyrus and CA3 regions of the hippocampus. Thus, it is important to know what types of spatial signals are present in these areas.

The postsubiculum is the region where head-direction cells were first discovered (Ranck Jr. 1985; Taube et al. 1990a), and cells in this region also show location correlates (Sharp 1996). Although the spatial tuning of postsubiculum cells is not as precise as that of CA1, it is similar to that of the subiculum (see below). Moreover, a significant proportion of location-specific cells in the postsubiculum and presubiculum are also sensitive to head direction (Sharp 1996; Cacucci et al. 2004). These place $x$ direction cells, modulated by theta (Cacucci et al. 2004), may be critical features of a path integration process, acting as internal units that allow an updating of position from one location to another based on the current directional heading of the rat. Other cells show tuning for head angular velocity and running speed, two properties that are necessary to allow self-motion information to update representations of location and head direction (Sharp 1996). Location-specific firing and place $\times$ direction firing were also found in the parasubiculum (Taube 1995; Hargreaves et al. 2005). In contrast, CA1 neurons show little directional tuning in pellet-chasing tasks (as opposed to tasks with stereotyped, constrained trajectories) (McNaughton et al. 1983; Muller et al. 1994; Markus et al. 1995). In the Hargreaves et al. (2005) study, the amount of spatial information conveyed by the parasubiculum neurons was indistinguishable from that of the MEC. Because these regions are located at a critical point in the processing loop of the hippocampus (i.e., they receive input from the subiculum and also project to the superficial layers of the entorhinal cortex as well as directly to the hippocampus proper), further investigation of the nature of the firing properties of these areas, in comparison with the properties of the hippocampus, will be crucial in identifying the types of processing performed by the hippocampus and the processing performed by these extrahippocampal areas.

\section{Septum}

The hippocampus has strong, bidirectional connections with the septal nuclei (Witter and Amaral 2004). Afferent connections arise from the medial septum, and these neurons display little spatial selectivity but are heavily modulated by the theta rhythm (Zhou et al. 1999). Projections from the hippocampus arrive primarily in the lateral septum, which contains spatially tuned neurons (Zhou et al. 1999; Leutgeb and Mizumori 2002), although spatial specificity is, on average, less precise than CA1 place cells. Unlike the postrhinal and perirhinal cortex, the spatial firing of lateral septal cells is reproducible across recording sessions in the same environment. Lateral septal neurons initially fire differently in a familiar and novel environment, but the representations appear to become more similar with repeated exposures to the novel environment (Leutgeb and Mizumori 2002). Thus, lateral septal cells may initially form context-specific representations, similar to the output from CA1, but it is unclear why (and under what conditions) the septal representation may generalize the two environments with repeated exposures.

\section{Subiculum}

The subiculum is a major output structure of the hippocampus. It receives its major input from CA1 and projects to the deep layers of the EC as well as to the septum (among other projections). Although the subiculum is considered part of the hippocampal formation, it has not been investigated as extensively as CA3 and CA1, either in terms of spatial or nonspatial properties (e.g., Sharp and Green 1994; Deadwyler and Hampson 2004). Subiculum neurons show a clear spatial selectivity, although their firing fields are not as precise as CA1 place fields. They tend to fire at a high rate throughout much of the environment, but with robust spatial peaks in the firing (Sharp and Green 1994; Martin and Ono 2000). A few examples of subicular "place fields" from the work of Sharp and colleagues (Sharp and Green 1994; Sharp 1999) demonstrate multipeaked firing that is reminiscent of the grid-cell phenomenon of the MEC. The spacing between the peaks is too large relative to the size of the recording environment to determine from these publications whether these were true grid cells. Because the MEC sends a direct projection to the 
subiculum, it would not be surprising to find a subset of grid cells (or similar cells) in the subiculum.

Perhaps the most important characteristic of subiculum spatial firing is that there appears to be little remapping of the subiculum representation, compared with CA1 (Sharp 1997, 1999). In experiments in which the same cells were recorded in cylindrical and square chambers, hippocampal place cells tended to form separate representations of each environment, whereas the subiculum represented each environment similarly. That is, some CA1 place cells had firing fields in only the cylinder or the square and were silent in the other chamber. In contrast, all but one of the subiculum cells fired at similar rates in both chambers, and the spatial firing fields of the cells were similar in both environments (Sharp 1997). Similar results were obtained when the cells were recorded in a small and large rectangular apparatus; whereas CA1 place cells tended to remap, subiculum place fields tended to expand/contract their firing fields along with the apparatus (Sharp 1999) (see also Muller and Kubie 1987). Sharp suggested that the subiculum represents a "universal map" utilized in all environments, perhaps for path integration, whereas the CA3 and CA1 place cells create context-specific maps used to distinguish different environments. In support of this role, subiculum cells, like CA1 place cells, also are sensitive to movement speed (Martin and Ono 2000; Anderson and O'Mara 2004) and head direction (Sharp and Green 1994), two signals that are necessary to properly update a representation of location based on selfmotion (McNaughton et al. 1996; Samsonovich and McNaughton 1997).

Cells in the distal portion of the subiculum (i.e., further from the CA1-subiculum border along the transverse axis of the hippocampus) show spatial selectivity that is slightly more precise than cells in the proximal (i.e., closer to CA1) portion of the subiculum (Sharp and Green 1994). This finding is consistent with the finding that the MEC, which projects to the distal subiculum, has more precise spatial tuning than the LEC, which projects to the proximal subiculum (Hargreaves et al. 2005). Although the proximal subiculum has more specific spatial tuning than LEC, this spatial signal may arise from its inputs from CA1. In addition, ventral subiculum appears to show little spatial selectivity in a pellet-chasing task (Hargreaves et al. 2005), consistent with the lack of high-resolution spatial tuning seen in the ventrolateral band of the MEC and the ventral hippocampus (Jung et al. 1994; Fyhn et al. 2004; Maurer et al. 2006). On the other hand, Phillips and Eichenbaum (1998) demonstrated spatial tuning in the ventral subiculum on a 4-arm maze, suggesting that the spatial tuning of ventral subiculum cells may depend on the types of cues or behaviors associated with the experimental task.

\section{Remapping from MEC to CAl to subiculum}

As mentioned above, both the subiculum and the MEC do not change their spatial representations under conditions where CA1 place fields readily "remap." Although some cells in the subiculum may remap under double-rotation conditions (Phillips and Eichenbaum 1998) or when environmental boundaries are removed (Lever et al. 2005), evidently the threshold for remapping in the subiculum is higher than that for CA1. If one of the major functions of the hippocampus is to create independent, contextspecific representations of an environment based on the potentially overlapping spatial input and nonspatial cues derived from the MEC and LEC, respectively (Redish 1999), it is surprising that the subiculum, which is a major output target of CA1, does not remap when its CA1 inputs have remapped. Presumably, the spatial tuning of the subiculum neurons is derived under these conditions from the direct entorhinal projections to the subiculum, with a minor role of the CA1 inputs. (A similar conundrum ap- plies to the relationship between CA3 and CA1 place fields. Under some conditions, the CA3 network appears to perform a pattern completion/generalization or a pattern separation operation, yet CA1 appears to "ignore" this input from CA3 and creates representations that are presumably more tightly correlated with the changes in its entorhinal inputs [Guzowski et al. 2004]). There are a number of possible explanations for why the subiculum does not remap when its CA1 afferents remap. (1) Because the major subcortical output of CA3 is to the lateral septum (Witter and Amaral 2004), which was shown to remap (at least initially) in a novel environment (Leutgeb and Mizumori 2002), it is possible that the CA3-septum pathway plays a critical role in context-dependent representations during online behavior through its connections with hypothalamic nuclei involved in arousal and behavioral activation (Risold 2004). (2) Hippocampal remapping may not be important in governing the rat's ongoing behavior (Jeffery et al. 2003). Rather, online behavior may be governed by interactions between the context-independent firing properties of the EC and subiculum, with little input from CA1. Under this scenario, the role of hippocampal processing through the DG-CA3-CA1 loop may be more important during periods of memory consolidation, perhaps during sleep, where context-specific representations stored in the hippocampus during behavior may guide the long-term consolidation of contextspecific memories in the neocortex (Marr 1971; Buzsaki 1989; Wilson and McNaughton 1994; McClelland et al. 1995). (3) Most experiments that have studied remapping in the MEC and subiculum have not utilized tasks that are strongly dependent on the hippocampus or that have large memory demands. Perhaps the direct EC-subiculum connections can support behavior under conditions of low memory demand, whereas the CA3-CA1 input is necessary for tasks that require specific associative memory operations, such as pattern separation or completion (Kesner et al. 2000). If one were to record the subiculum under these conditions, it is possible that the subiculum might remap more readily. (4) Finally, the effect of CA1 remapping in the subiculum may be evident in more subtle ways than changes in firing locations, such as changes in firing rate, ensemble coordination, or so forth (Leutgeb et al. 2005). Clearly, understanding the relationship between remapping in the CA3-CA1 pyramidal layers and in the MEC input and subiculum output layers is a critically important step in understanding the functional role of the spatial representations in these regions.

\section{Other brain systems}

Hippocampus-dependent tasks always require additional brain systems. Sensory and motor systems are necessary to process information about environmental conditions and to generate motor outputs to solve the task. Other learning systems are also necessary. For example, the cerebellum, prefrontal cortex, and hippocampus are all required for trace eyelid conditioning (Woodruff-Pak et al. 1985; Moyer et al. 1990; Weible et al. 2000). It is thus of interest to determine whether systems outside of the parahippocampal region show spatially selective firing. Investigations along these lines have concentrated on the striatum, the prefrontal cortex, the posterior cortex, and the superior colliculus.

\section{Striatum}

The striatum is often characterized as being involved in response or habit learning (Packard and Knowlton 2002). There appears to be a time-dependent relationship between hippocampal and striatal contributions in certain spatial tasks (Packard and McGaugh 1996; Chang and Gold 2003). For example, rats initially 
use a hippocampus-dependent, spatial strategy (Go to the arm at location X) to solve a T-maze task, but over time switch to a striatum-dependent, response strategy (Make a right turn at the choice point). Dorsal striatum contains head-direction cells (Wiener 1993; Mizumori et al. 2000; Ragozzino et al. 2001) and cells that are correlated with different aspects of motion. In addition, a number of studies have found location-specific firing in a subset of striatal cells, but these representations are often modulated by behavioral or reward correlates. For example, when rats performed a task where they had to run from the center of a square chamber to each corner for a reward, dorsal striatal cells responded differentially during runs in each quadrant, but only when the rats performed certain sequences of behavior in that quadrant (the cells did not respond to the same behavior in other quadrants) (Wiener 1993). Striatal cells also correlate with the location (left or right) of a response lever in a delayed match-tosample task (Chang et al. 2002). Striatal place-correlated cells respond to contextual changes in ways that are similar to hippocampal place cells. When proximal and distal landmarks are rotated relative to each other or are scrambled, both CA1 place cells and striatal place-related cells change their firing fields, becoming silent, developing new fields, or changing their firing locations (Yeshenko et al. 2004). Striatal place-related cells appear to be more sensitive to darkness than CA1 place cells, however, as the majority of them change their firing patterns when the lights are turned off, whereas CA1 place fields are typically robust to changes in illumination (McNaughton et al. 1989; Quirk et al. 1990; Markus et al. 1994; Mizumori et al. 2000). Thus, striatal place-related cells may be more sensitive to visual cues than are CA1 place cells (Mizumori et al. 2000).

On a multiple-T-maze, in which a series of left and right choice points must be navigated properly, dorsal striatum cells displayed spatial firing fields at specific locations throughout the entire extent of the maze (Schmitzer-Torbert and Redish 2004). Cells that were responsive to reward on the maze distinguished between the two reward locations. Similar results were shown in the early stages of learning in a T-maze task, in which rats had to learn to associate a particular tone heard on the stem of the maze with a particular behavioral response at the choice point (left or right turn) (Jog et al. 1999). Initially, individual striatal cells fired at specific locations, but the population representation covered the entire T-maze. As the rats learned to perform the task at a high level of proficiency (i.e., the task became like a habit), the cells changed their firing locations to represent mostly the start location of the trial and the end location of the trial. Interestingly, when the response was extinguished, the cells began to represent the entire maze again, and then reverted quickly back to the more selective firing at the start and end during relearning (Barnes et al. 2005). These results suggest that the spatial-firing properties of striatal neurons may be more directly tied to task parameters than CA1 place cells.

The ventral striatum and nucleus accumbens have also received special attention based on their relationship to the ventral tegmental area and connections with the limbic system. Similar to the dorsal striatum, a subset of ventral striatum and nucleus accumbens neurons show spatial sensitivity (Shibata et al. 2001; Mulder et al. 2005), in some case quite specific (Lavoie and Mizumori 1994). In many of these studies, the spatial sensitivity was modulated by reward anticipation or approach (Martin and Ono 2000; Shibata et al. 2001). In a +-maze, nucleus accumbens and ventromedial caudate neurons fired throughout a particular behavioral sequence of the task (e.g., firing along the entire trajectory from one start position to the goal position) (Mulder et al. 2004). These studies have been interpreted as reflecting the hypothesized role of the nucleus accumbens and ventral striatum in integrating spatial and reward information with the motor sys- tem to produce adaptive behavioral outputs, perhaps in association with the offline "memory reactivation" hypothesized to occur during sharp wave-ripple activity in the hippocampus (Pennartz et al. 2004).

\section{Prefrontal cortex}

The prefrontal cortex (PFC) is particularly associated with the ventral hippocampus, where place fields are not as specific as dorsal hippocampus (Jung et al. 1994; Maurer et al. 2006). Consistent with this dissociation, in random foraging or pelletchasing tasks, PFC neurons do not show spatial selectivity (Poucet 1997; Jung et al. 1998; Gemmell et al. 2002). However, when a goal-finding task was incorporated in the same environment, some PFC cells fired at one of three locations, i.e., the goal zone, the "feeder" zone (where the food reward landed after falling from the overhead dispenser), and the single cue card that provided a salient orientation cue to the rat (Hok et al. 2005). Interestingly, the selective firing was maintained even when the rat was performing the pellet-chasing phase of the task to find the reward, in between the goal-searching trials. On 8-arm maze tasks, few cells display spatially selective firing; most of the correlates of PFC neurons appear to be related to behavior, rather than to place (Jung et al. 1998; Pratt and Mizumori 2001). However, clear interactions between hippocampus and PFC have been demonstrated in terms of PFC neurons phase-locking to the hippocampal theta rhythm (Hyman et al. 2005; Jones and Wilson 2005), in tasks where PFC neurons demonstrate large place fields (Jones and Wilson 2005). This phase-locking is most prevalent during segments of the task that require larger memory demands, suggesting that the PFC and hippocampus work as a temporally coordinated system during periods where behavioral performance requires accurate memory processing.

\section{Parietal cortex, retrosplenial cortex, and superior colliculus}

In the visual system, two regions that are particularly associated with spatial processing are the posterior parietal cortex (visuospatial processing) and the superior colliculus (orienting to visual stimuli). In rodents, the existence of head-direction cells in the retrosplenial cortex has implicated this region in spatial processing. However, little evidence exists in rats for location-specific firing in the posterior cortex or retrosplenial cortex (Chen et al. 1994a,b; McNaughton et al. 1994). In behaving rats, many cells in these regions are correlated with various aspects of movement (e.g., right or left turns) (McNaughton et al. 1994), although some cells in retrosplenial cortex may correlate with the approach to a particular location (Cho and Sharp 2001). Interestingly, in area OC2M, neurons demonstrate an allocentric representation of the location of auditory cues (Nakamura 1999), showing that although spatial location is an important function of these regions, it does not produce selectivity for the spatial location of the animal. Finally, recordings of the superior colliculus revealed a number of spatial, nonspatial, and directional cells; however, the spatial firing was not stable across sessions, suggesting that these responses were not robust representations of the rat's location in the environment (Cooper et al. 1998).

\section{Concluding comments}

Just as spatial location is not the only firing correlate of rat hippocampal neurons (although it may be the required baseline correlate), location-related firing is not the exclusive domain of the hippocampus, as other brain regions show various degrees of spatially selective firing. Nonetheless, there are important differences among these regions. The most specific place fields are 
found in the dentate gyrus, CA3, and CA1 regions of the hippocampus. These cells tend to fire in small, restricted locations, with virtually no firing outside of the place field. Thus, the hippocampus provides a sparse representation of the environment, compared with the coarser tuning of other areas. Locationrelated firing occurs as a subset of response types in these other brain areas, intermixed with cells that have nonspatial firing correlates. In contrast, all hippocampal principal neurons are thought to be potential place cells (at least in dorsal hippocampus); even though only a subset of them fire in a particular environment, the other cells that are silent can show place fields in a different environment. Although hippocampal place fields can be modulated by nonspatial factors, such nonspatial modulation does not appear to be critical to demonstrate the place-specific firing. In contrast, the spatial firing in extrahippocampal regions may be more readily modifiable by nonspatial factors (e.g., reward, behavior), and may require the presence of these factors. These differences suggest that, compared with these other brain regions, the hippocampus plays a particularly dedicated role to spatial representations.

What is the function of the spatially tuned firing of cells in extrahippocampal areas? One possibility is that place-related response properties are generated in these areas as part of specific computations performed by the areas. For example, striatal place cells may form independent of hippocampal place cells as part of the information processing required of stratum-dependent tasks. In the MEC, grid cells are present even when the hippocampus proper is lesioned, demonstrating that this property is largely independent of the hippocampus (Fyhn et al. 2004). In other cases, location-specific firing in these regions may occur as a direct consequence of the firing of hippocampal place cells. Perhaps these cells serve an important role in calibrating the activity of multiple brain representations to the specific spatiotemporal framework of ongoing behavior. Just as different sensory representations need to be adjusted and calibrated to produce unified percepts (e.g., auditory and visual maps of space in the barn owl) (Brainard and Knudsen 1998), perhaps the different brain systems involved in performance of behavioral tasks require calibration of their representations to a consistent spatial and temporal framework (O'Keefe and Nadel 1978). The spatially selective neurons in these regions may be the mediators by which contextspecific firing in the hippocampus organizes these representations (perhaps with the help of head direction cells that are also found in numerous brain regions) and adds context-specificity to the output of these regions. Certainly, more research is required to address these issues. The spatial tuning of neurons in these areas must be more thoroughly explored as well as the interactions between the spatial and nonspatial properties of these regions. Hippocampal and entorhinal lesions will help determine the extent to which these firing patterns depend on an intact hippocampus and how much they are generated independent of the hippocampus. By contrasting the spatial firing properties of these regions with hippocampal regions, a clearer picture may emerge of the fundamental processing and computations that are performed by the hippocampus; how the output of hippocampal processing may be used to facilitate or coordinate the processing of other brain areas; and how the representations that are present in the hippocampal input structures allow the hippocampus to create the complex, dynamic spatial representations that are displayed by place fields. Understanding the computational principles that underlie these transformations in the rat brain holds promise for ultimately elucidating similar principles of the human hippocampus, which presumably receives more complex input representations than the rat hippocampus, but performs similar computations that are somehow necessary for the types of memory that are lost in medial temporal lobe amnesia.

\section{Acknowledgments}

The author's research is supported by grants R01 NS039456, K02 MH063297, and P01 NS038310 from the Public Health Service and by grant IBN-0344213 from the National Science Foundation.

\section{References}

Anderson, M.I. and O'Mara, S.M. 2004. Responses of dorsal subicular neurons of rats during object exploration in an extended environment. Exp. Brain Res. 159: 519-529.

Barnes, C.A., McNaughton, B.L., Mizumori, S.J., Leonard, B.W., and Lin, L.H. 1990. Comparison of spatial and temporal characteristics of neuronal activity in sequential stages of hippocampal processing. Prog. Brain Res. 83: 287-300.

Barnes, T.D., Kubota, Y., Hu, D., Jin, D.Z., and Graybiel, A.M. 2005. Activity of striatal neurons reflects dynamic encoding and recoding of procedural memories. Nature 437: 1158-1161.

Berger, T.W., Alger, B., and Thompson, R.F. 1976. Neuronal substrate of classical conditioning in the hippocampus. Science 192: 483-485.

Bower, M.R., Euston, D.R., and McNaughton, B.L. 2005. Sequential-context-dependent hippocampal activity is not necessary to learn sequences with repeated elements. J. Neurosci. 25: $1313-1323$.

Brainard, M.S. and Knudsen, E.I. 1998. Sensitive periods for visual calibration of the auditory space map in the barn owl optic tectum. J. Neurosci. 18: 3929-3942.

Burwell, R.D. 2000. The parahippocampal region: Corticocortical connectivity. Ann. N. Y. Acad. Sci. 911: 25-42.

Burwell, R.D. and Hafeman, D.M. 2003. Positional firing properties of postrhinal cortex neurons. Neuroscience 119: 577-588.

Burwell, R.D., Witter, M.P., and Amaral, D.G. 1995. Perirhinal and postrhinal cortices of the rat: A review of the neuroanatomical literature and comparison with findings from the monkey brain. Hippocampus 5: 390-408.

Burwell, R.D., Shapiro, M.L., O’Malley, M.T., and Eichenbaum, H. 1998. Positional firing properties of perirhinal cortex neurons. Neuroreport 9: 3013-3018.

Buzsaki, G. 1989. Two-stage model of memory trace formation: A role for "noisy" brain states. Neuroscience 31: 551-570.

Cacucci, F., Lever, C., Wills, T.J., Burgess, N., and O'Keefe, J. 2004. theta-modulated place-by-direction cells in the hippocampal formation in the rat. J. Neurosci. 24: 8265-8277.

Chang, Q. and Gold, P.E. 2003. Switching memory systems during learning: Changes in patterns of brain acetylcholine release in the hippocampus and striatum in rats. J. Neurosci. 23: 3001-3005.

Chang, J.Y., Chen, L., Luo, F., Shi, L.H., and Woodward, D.J. 2002. Neuronal responses in the frontal cortico-basal ganglia system during delayed matching-to-sample task: Ensemble recording in freely moving rats. Exp. Brain Res. 142: 67-80.

Chen, L.L., Lin, L.H., Green, E.J., Barnes, C.A., and McNaughton, B.L. 1994a. Head-direction cells in the rat posterior cortex. I. Anatomical distribution and behavioral modulation. Exp. Brain Res. 101: 8-23.

Chen, L.L., Lin, L.H., Barnes, C.A., and McNaughton, B.L. 1994b. Head-direction cells in the rat posterior cortex. II. Contributions of visual and ideothetic information to the directional firing. Exp. Brain Res. 101: 24-34.

Cho, J. and Sharp, P.E. 2001. Head direction, place, and movement correlates for cells in the rat retrosplenial cortex. Behav. Neurosci. 115: $3-25$.

Cohen, N.J. and Eichenbaum, H. 1993. Memory, amnesia, and the hippocampal system. MIT Press, Cambridge, MA.

Cooper, B.G., Miya, D.Y., and Mizumori, S.J. 1998. Superior colliculus and active navigation: Role of visual and non-visual cues in controlling cellular representations of space. Hippocampus 8: $340-372$.

Davachi, L., Mitchell, J.P., and Wagner, A.D. 2003. Multiple routes to memory: Distinct medial temporal lobe processes build item and source memories. Proc. Natl. Acad. Sci. 100: 2157-2162.

Deadwyler, S.A. and Hampson, R.E. 2004. Differential but complementary mnemonic functions of the hippocampus and subiculum. Neuron 42: 465-476.

Dolorfo, C.L. and Amaral, D.G. 1998. Entorhinal cortex of the rat: Topographic organization of the cells of origin of the perforant path projection to the dentate gyrus. J. Comp. Neurol. 398: 25-48.

Eichenbaum, H. 2004. Hippocampus: Cognitive processes and neural representations that underlie declarative memory. Neuron 44: $109-120$.

Ekstrom, A.D., Kahana, M.J., Caplan, J.B., Fields, T.A., Isham, E.A.,

\section{Learning \& Memory}


Newman, E.L., and Fried, I. 2003. Cellular networks underlying human spatial navigation. Nature 425: 184-188.

Etienne, A.S. 1992. Navigation of a small mammal by dead reckoning and local cues. Curr. Dir. Psychol. Sci. 1: 48-52.

Ferbinteanu, J. and Shapiro, M.L. 2003. Prospective and retrospective memory coding in the hippocampus. Neuron 40: 1227-1239.

Frank, L.M., Brown, E.N., and Wilson, M. 2000. Trajectory encoding in the hippocampus and entorhinal cortex. Neuron 27: 169-178.

Frank, L.M., Stanley, G.B., and Brown, E.N. 2004. Hippocampal plasticity across multiple days of exposure to novel environments. $J$. Neurosci. 24: 7681-7689.

Fuhs, M.C. and Touretzky, D.S. 2006. A spin glass model of path integration in rat medial entorhinal cortex. J. Neurosci. 26: $4266-4276$.

Fyhn, M., Molden, S., Witter, M.P., Moser, E.I., and Moser, M.B. 2004. Spatial representation in the entorhinal cortex. Science 305: $1258-1264$

Gaffan, D. 1998. Idiothetic input into object-place configuration as the contribution to memory of the monkey and human hippocampus: A review. Exp. Brain Res. 123: 201-209.

Gallistel, C.R. 1990 The organization of learning. MIT Press. Cambridge, MA.

Gemmell, C., Anderson, M., and O’Mara, S.M. 2002. Deep layer prefrontal cortex unit discharge in a cue-controlled open-field environment in the freely-moving rat. Behav. Brain Res. 133: 1-10.

Guzowski, J.F., Knierim, J.J., and Moser, E.I. 2004. Ensemble dynamics of hippocampal regions CA3 and CA1. Neuron 44: 581-584.

Hafting, T., Fyhn, M., Molden, S., Moser, M.B., and Moser, E.I. 2005. Microstructure of a spatial map in the entorhinal cortex. Nature 436: 801-806.

Hargreaves, E.L., Rao, G., Lee, I., and Knierim, J.J. 2005. Major dissociation between medial and lateral entorhinal input to dorsal hippocampus. Science 308: 1792-1794.

Hok, V., Save, E., Lenck-Santini, P.P., and Poucet, B. 2005. Coding for spatial goals in the prelimbic/infralimbic area of the rat frontal cortex. Proc. Natl. Acad. Sci. 102: 4602-4607.

Huxter, J., Burgess, N., and O'Keefe, J. 2003. Independent rate and temporal coding in hippocampal pyramidal cells. Nature 425: 828-832.

Hyman, J.M., Zilli, E.A., Paley, A.M., and Hasselmo, M.E. 2005. Medial prefrontal cortex cells show dynamic modulation with the hippocampal theta rhythm dependent on behavior. Hippocampus 15: 739-749.

Jeffery, K.J., Gilbert, A., Burton, S., and Strudwick, A. 2003. Preserved performance in a hippocampal-dependent spatial task despite complete place cell remapping. Hippocampus 13: 175-189.

Jensen, O. and Lisman, J.E. 2005. Hippocampal sequence-encoding driven by a cortical multi-item working memory buffer. Trends Neurosci. 28: 67-72.

Jog, M.S., Kubota, Y., Connolly, C.I., Hillegaart, V., and Graybiel, A.M 1999. Building neural representations of habits. Science 286: $1745-1749$.

Jones, M.W. and Wilson, M.A. 2005. Theta rhythms coordinate hippocampal-prefrontal interactions in a spatial memory task. PLoS Biol. 3: e402.

Jung, M.W., Wiener, S.I., and McNaughton, B.L. 1994. Comparison of spatial firing characteristics of units in dorsal and ventral hippocampus of the rat. J. Neurosci. 14: 7347-7356.

Jung, M.W., Qin, Y., McNaughton, B.L., and Barnes, C.A. 1998. Firing characteristics of deep layer neurons in prefrontal cortex in rats performing spatial working memory tasks. Cereb. Cortex 8: 437-450.

Kesner, R.P., Gilbert, P.E., and Wallenstein, G.V. 2000. Testing neural network models of memory with behavioral experiments. Curr. Opin. Neurobiol. 10: 260-265.

Kloosterman, F., Van Haeften, T., Witter, M.P., and Lopes, D.S.F. 2003. Electrophysiological characterization of interlaminar entorhinal connections: An essential link for re-entrance in the hippocampal-entorhinal system. Eur. J. Neurosci. 18: 3037-3052.

Knierim, J.J., Kudrimoti, H.S., and McNaughton, B.L. 1995. Place cells, head direction cells, and the learning of landmark stability. $J$. Neurosci. 15: 1648-1659.

Lavoie, A.M. and Mizumori, S.J. 1994. Spatial, movement- and reward-sensitive discharge by medial ventral striatum neurons of rats. Brain Res. 638: 157-168.

Lee, I., Rao, G., and Knierim, J.J. 2004. A double dissociation between hippocampal subfields: Differential time course of CA3 and CA1 place cells for processing changed environments. Neuron 42: $803-815$.

Lenck-Santini, P.P., Save, E., and Poucet, B. 2001. Place-cell firing does not depend on the direction of turn in a Y-maze alternation task. Eur. J. Neurosci. 13: 1055-1058.

Leutgeb, S. and Mizumori, S.J. 2002. Context-specific spatial representations by lateral septal cells. Neuroscience 112: 655-663.

Leutgeb, S., Leutgeb, J.K., Barnes, C.A., Moser, E.I., McNaughton, B.L., and Moser, M.B. 2005. Independent codes for spatial and episodic memory in hippocampal neuronal ensembles. Science 309: 619-623.

Lever, C., Burton, S., Wills, T.J., Burgess, N., and O'Keefe, J. 2005. Dorsal subicular and CA1 hippocampal neurons react differently to environmental novelty. Abstract Viewer/Itinerary Planner, Program No. 72.8. Society for Neuroscience, Washington, D.C.

Levy, W.B. 1996. A sequence predicting CA3 is a flexible associator that learns and uses context to solve hippocampal-like tasks. Hippocampus 6: $579-590$.

Lindquist, D.H., Jarrard, L.E., and Brown, T.H. 2004. Perirhinal cortex supports delay fear conditioning to rat ultrasonic social signals. $J$. Neurosci. 24: 3610-3617.

Ludvig, N., Tang, H.M., Gohil, B.C., and Botero, J.M. 2004. Detecting location-specific neuronal firing rate increases in the hippocampus of freely-moving monkeys. Brain Res. 1014: 97-109.

Markus, E.J., Barnes, C.A., McNaughton, B.L., Gladden, V.L., and Skaggs, W.E. 1994. Spatial information content and reliability of hippocampal CA1 neurons: Effects of visual input. Hippocampus 4: $410-421$.

Markus, E.J., Qin, Y.L., Leonard, B., McNaughton, B.L., and Barnes, C.A. 1995. Interactions between location and task affect the spatial and directional firing of hippocampal neurons. J. Neurosci. 15: 7079-7094.

Marr, D. 1971. Simple memory: A theory for archicortex. Philos. Trans. R. Soc. Lond. B Biol. Sci. 262: 23-81.

Martin, P.D. and Ono, T. 2000. Effects of reward anticipation, reward presentation, and spatial parameters on the firing of single neurons recorded in the subiculum and nucleus accumbens of freely moving rats. Behav. Brain Res. 116: 23-38.

Maurer, A.P., Vanrhoads, S.R., Sutherland, G.R., Lipa, P., and McNaughton, B.L. 2006. Self-motion and the origin of differential spatial scaling along the septo-temporal axis of the hippocampus. Hippocampus 15: 841-852.

McClelland, J.L., McNaughton, B.L., and O'Reilly, R.C. 1995. Why there are complementary learning systems in the hippocampus and neocortex: Insights from the successes and failures of connectionist models of learning and memory. Psychol. Rev. 102: 419-457.

McEchron, M.D. and Disterhoft, J.F. 1997. Sequence of single neuron changes in CA1 hippocampus of rabbits during acquisition of trace eyeblink conditioned responses. J. Neurophysiol. 78: 1030-1044.

McNaughton, B.L. and Morris, R.G.M. 1987. Hippocampal synaptic enhancement and information storage within a distributed memory system. Trends Neurosci. 10: 408-415.

McNaughton, B.L., Barnes, C.A., and O'Keefe, J. 1983. The contributions of position, direction, and velocity to single unit activity in the hippocampus of freely-moving rats. Exp. Brain Res. 52: 41-49.

McNaughton, B.L., Leonard, B., and Chen, L.L. 1989. Cortical-hippocampal interactions and cognitive mapping: A hypothesis based on reintegration of the parietal and inferotemporal pathways for visual processing. Psychobiology 17: 230-235.

McNaughton, B.L., Mizumori, S.J., Barnes, C.A., Leonard, B.J., Marquis, M., and Green, E.J. 1994. Cortical representation of motion during unrestrained spatial navigation in the rat. Cereb. Cortex 4: 27-39.

McNaughton, B.L., Barnes, C.A., Gerrard, J.L., Gothard, K., Jung, M.W. Knierim, J.J., Kudrimoti, H., Qin, Y., Skaggs, W.E., Suster, M., et al. 1996. Deciphering the hippocampal polyglot: The hippocampus as a path integration system. J. Exp. Biol. 199: 173-185.

Mehta, M.R., Barnes, C.A., and McNaughton, B.L. 1997. Experience-dependent, asymmetric expansion of hippocampal place fields. Proc. Natl. Acad. Sci. 94: 8918-8921.

Mittelstaedt, M.L. and Mittelstaedt, H. 1980. Homing by path integration in a mammal. Naturwissenschaften 67: 566-567.

Mizumori, S.J., Ward, K.E., and Lavoie, A.M. 1992. Medial septal modulation of entorhinal single unit activity in anesthetized and freely moving rats. Brain Res. 570: $188-197$.

Mizumori, S.J.Y., Ragozzino, K.E., and Cooper, B.G. 2000. Location and head direction representation in the dorsal striatum of rats. Psychobiology 28: 441-462.

Moita, M.A., Rosis, S., Zhou, Y., LeDoux, J.E., and Blair, H.T. 2003. Hippocampal place cells acquire location-specific responses to the conditioned stimulus during auditory fear conditioning. Neuron 37: 485-497.

Moyer, J.R.J., Deyo, R.A., and Disterhoft, J.F. 1990. Hippocampectomy disrupts trace eye-blink conditioning in rabbits. Behav. Neurosci. 104: $243-252$

Mulder, A.B., Tabuchi, E., and Wiener, S.I. 2004. Neurons in hippocampal afferent zones of rat striatum parse routes into multi-pace segments during maze navigation. Eur. J. Neurosci. 19: 1923-1932.

Mulder, A.B., Shibata, R., Trullier, O., and Wiener, S.I. 2005. Spatially 
selective reward site responses in tonically active neurons of the nucleus accumbens in behaving rats. Exp. Brain Res. 163: 32-43.

Muller, R.U. and Kubie, J.L. 1987. The effects of changes in the environment on the spatial firing of hippocampal complex-spike cells. J. Neurosci. 7: 1951-1968.

Muller, R.U., Kubie, J.L., and Ranck Jr., J.B. 1987. Spatial firing patterns of hippocampal complex-spike cells in a fixed environment. $J$. Neurosci. 7: 1935-1950.

Muller, R.U., Bostock, E., Taube, J.S., and Kubie, J.L. 1994. On the directional firing properties of hippocampal place cells. J. Neurosci. 14: $7235-7251$.

Nadel, L., Wilner, J., and Kurtz, E.M. 1985. Cognitive maps and environmental context. In Context and learning (eds. P.D. Balsam and A. Tomie), pp. 385-406. Earlbaum, Hillsdale, N.J.

Nakamura, K. 1999. Auditory spatial discriminatory and mnemonic neurons in rat posterior parietal cortex. J. Neurophysiol. 82: $2503-2517$.

Nishijo, H., Ono, T., Eifuku, S., and Tamura, R. 1997. The relationship between monkey hippocampus place-related neural activity and action in space. Neurosci. Lett. 226: 57-60.

Norman, G. and Eacott, M.J. 2005. Dissociable effects of lesions to the perirhinal cortex and the postrhinal cortex on memory for context and objects in rats. Behav. Neurosci. 119: 557-566.

O'Keefe, J. 1976. Place units in the hippocampus of the freely moving rat. Exp. Neurol. 51: 78-109.

. 1999. Do hippocampal pyramidal cells signal non-spatial as well as spatial information? Hippocampus 9: 352-364.

O'Keefe, J. and Burgess, N. 2005. Dual phase and rate coding in hippocampal place cells: Theoretical significance and relationship to entorhinal grid cells. Hippocampus 15: 853-866.

O'Keefe, J. and Conway, D.H. 1978. Hippocampal place units in the freely moving rat: Why they fire where they fire. Exp. Brain Res. 31: 573-590.

O'Keefe, J. and Dostrovsky, J. 1971. The hippocampus as a spatial map: Preliminary evidence from unit activity in the freely-moving rat. Brain Res. 34: 171-175.

O'Keefe, J. and Nadel, L. 1978. The hippocampus as a cognitive map. Clarendon Press, Oxford, UK.

O'Keefe, J. and Speakman, A. 1987. Single unit activity in the rat hippocampus during a spatial memory task. Exp. Brain Res. 68: 1-27.

Packard, M.G. and Knowlton, B.J. 2002. Learning and memory functions of the basal ganglia. Annu. Rev. Neurosci. 25: 563-593.

Packard, M.G. and McGaugh, J.L. 1996. Inactivation of hippocampus or caudate nucleus with lidocaine differentially affects expression of place and response learning. Neurobiol. Learn. Mem. 65: 65-72.

Pennartz, C.M., Lee, E., Verheul, J., Lipa, P., Barnes, C.A., and McNaughton, B.L. 2004. The ventral striatum in off-line processing: Ensemble reactivation during sleep and modulation by hippocampal ripples. J. Neurosci. 24: 6446-6456.

Phillips, R.G. and Eichenbaum, H. 1998. Comparison of ventral subicular and hippocampal neuron spatial firing patterns in complex and simplified environments. Behav. Neurosci. 112: 707-713.

Poucet, B. 1997. Searching for spatial unit firing in the prelimbic area of the rat medial prefrontal cortex. Behav. Brain Res. 84: 151-159.

Pratt, W.E. and Mizumori, S.J. 2001. Neurons in rat medial prefrontal cortex show anticipatory rate changes to predictable differential rewards in a spatial memory task. Behav. Brain Res. 123: 165-183.

Quirk, G.J., Muller, R.U., and Kubie, J.L. 1990. The firing of hippocampal place cells in the dark depends on the rat's recent experience. J. Neurosci. 10: 2008-2017.

Quirk, G.J., Muller, R.U., Kubie, J.L., and Ranck Jr., J.B. 1992. The positional firing properties of medial entorhinal neurons: Description and comparison with hippocampal place cells. $J$. Neurosci. 12: 1945-1963.

Ragozzino, K.E., Leutgeb, S., and Mizumori, S.J. 2001. Dorsal striatal head direction and hippocampal place representations during spatial navigation. Exp. Brain Res. 139: 372-376.

Ranck Jr., J.B. 1985. Head direction cells in the deep cell layer of dorsal presubiculum in freely moving rats. In Electrical activity of archicortex (eds. G. Buzsaki and C.H. Vanderwolf), pp. 217-220. Akademiai Kiado, Budapest.

Redish, A.D. 1999. Beyond the cognitive map: From place cells to episodic memory. MIT Press, Cambridge, MA.

Risold, P.Y. 2004. The septal region. In The rat nervous system, 3rd ed (ed. G. Paxinos), pp. 605-632. Elsevier, Amsterdam.

Rolls, E.T. and Treves, A. 1998. Neural networks and brain function. Oxford University Press, Oxford, UK.

Rolls, E.T., Robertson, R.G., and Georges-Francois, P. 1997. Spatial view cells in the primate hippocampus. Eur. J. Neurosci. 9: 1789-1794.

Samsonovich, A. and McNaughton, B.L. 1997. Path integration and cognitive mapping in a continuous attractor neural network model. I. Neurosci. 17: 5900-5920.
Schmitzer-Torbert, N. and Redish, A.D. 2004. Neuronal activity in the rodent dorsal striatum in sequential navigation: Separation of spatial and reward responses on the multiple T task. J. Neurophysiol. 91: 2259-2272.

Scoville, W.B. and Milner, B. 1957. Loss of recent memory after bilateral hippocampal lesions. J. Neurol. Neurosurg. Psychiatry 12: 103-113.

Shapiro, M.L. and Eichenbaum, H. 1999. Hippocampus as a memory map: Synaptic plasticity and memory encoding by hippocampal neurons. Hippocampus 9: 365-384.

Sharp, P.E. 1996. Multiple spatial/behavioral correlates for cells in the rat postsubiculum: Multiple regression analysis and comparison to other hippocampal areas. Cereb. Cortex 6: 238-259. . 1997. Subicular cells generate similar spatial firing patterns in two geometrically and visually distinctive environments: Comparison with hippocampal place cells. Behav. Brain Res. 85: 71-92.

- 1999. Subicular place cells expand or contract their spatial firing pattern to fit the size of the environment in an open field but not in the presence of barriers: Comparison with hippocampal place cells. Behav. Neurosci. 113: 643-662.

Sharp, P.E. and Green, C. 1994. Spatial correlates of firing patterns of single cells in the subiculum of the freely moving rat. J. Neurosci. 14: 2339-2356.

Shibata, R., Mulder, A.B., Trullier, O., and Wiener, S.I. 2001. Position sensitivity in phasically discharging nucleus accumbens neurons of rats alternating between tasks requiring complementary types of spatial cues. Neuroscience 108: 391-411.

Squire, L.R. 1987. Memory and brain. Oxford University Press, Oxford, UK.

Sutherland, R.J. and Rudy, J.W. 1989. Configural association theory: The role of the hippocampal formation in learning, memory, and amnesia. Psychobiology 17: 129-144.

Suzuki, W.A., Miller, E.K., and Desimone, R. 1997. Object and place memory in the macaque entorhinal cortex. J. Neurophysiol. 78: $1062-1081$.

Taube, J.S. 1995. Place cells recorded in the parasubiculum of freely moving rats. Hippocampus 5: 569-583.

Taube, J.S., Muller, R.U., and Ranck Jr., J.B. 1990a. Head-direction cells recorded from the postsubiculum in freely moving rats. II. Effects of environmental manipulations. J. Neurosci. 10: 436-447. . 1990b. Head-direction cells recorded from the postsubiculum in freely moving rats. I. Description and quantitative analysis. $J$. Neurosci. 10: $420-435$.

Teyler, T.J. and DiScenna, P. 1985. The role of the hippocampus in memory: A hypothesis. Neurosci. Biobehav. Rev. 9: 377-389.

Tolman, E.C. 1948. Cognitive maps in rats and men. Psychol. Bull. 55: $189-208$.

Van Haeften, T., Baks, T.B., Goede, P.H., Wouterlood, F.G., and Witter, M.P. 2003. Morphological and numerical analysis of synaptic interactions between neurons in deep and superficial layers of the entorhinal cortex of the rat. Hippocampus 13: 943-952.

Wallenstein, G.V., Eichenbaum, H., and Hasselmo, M.E. 1998. The hippocampus as an associator of discontiguous events. Trends Neurosci. 21: 317-323.

Wan, H., Aggleton, J.P., and Brown, M.W. 1999. Different contributions of the hippocampus and perirhinal cortex to recognition memory. $J$. Neurosci. 19: 1142-1148.

Weible, A.P., McEchron, M.D., and Disterhoft, J.F. 2000. Cortical involvement in acquisition and extinction of trace eyeblink conditioning. Behav. Neurosci. 114: 1058-1067.

Wiebe, S.P. and Staubli, U.V. 1999. Dynamic filtering of recognition memory codes in the hippocampus. J. Neurosci. 19: 10562-10574.

Wiener, S.I. 1993. Spatial and behavioral correlates of striatal neurons in rats performing a self-initiated navigation task. J. Neurosci. 13: $3802-3817$

Wilson, M.A. and McNaughton, B.L. 1994. Reactivation of hippocampal ensemble memories during sleep. Science 265: 676-679.

Witter, M.P. and Amaral, D.G. 2004. Hippocampal formation. In The rat nervous system, 3rd ed. (ed. G. Paxinos), pp. 635-704. Elsevier, Amsterdam.

Witter, M.P., Wouterlood, F.G., Naber, P.A., and Van Haeften, T. 2000. Anatomical organization of the parahippocampal-hippocampal network. Ann. N. Y. Acad. Sci. 911: 1-24.

Wood, E.R., Dudchenko, P.A., and Eichenbaum, H. 1999. The global record of memory in hippocampal neuronal activity. Nature 397: 613-616.

Wood, E.R., Dudchenko, P.A., Robitsek, R.J., and Eichenbaum, H. 2000. Hippocampal neurons encode information about different types of memory episodes occurring in the same location. Neuron 27: 623-633.

Woodruff-Pak, D.S., Lavond, D.G., and Thompson, R.F. 1985. Trace conditioning: Abolished by cerebellar nuclear lesions but not lateral 
cerebellar cortex aspirations. Brain Res. 348: 249-260.

Yeshenko, O., Guazzelli, A., and Mizumori, S.J. 2004.

Context-dependent reorganization of spatial and movement

representations by simultaneously recorded hippocampal and striatal

neurons during performance of allocentric and egocentric tasks.

Behav. Neurosci. 118: 751-769.

Zhou, T.L., Tamura, R., Kuriwaki, J., and Ono, T. 1999. Comparison of medial and lateral septal neuron activity during performance of spatial tasks in rats. Hippocampus 9: 220-234.

Zhu, X.O., Brown, M.W., and Aggleton, J.P. 1995. Neuronal signalling of information important to visual recognition memory in rat rhinal and neighbouring cortices. Eur. J. Neurosci. 7: 753-765.

Zironi, I., Iacovelli, P., Aicardi, G., Liu, P., and Bilkey, D.K. 2001. Prefrontal cortex lesions augment the location-related firing properties of area TE/perirhinal cortex neurons in a working memory task. Cereb. Cortex 11: 1093-1100. 


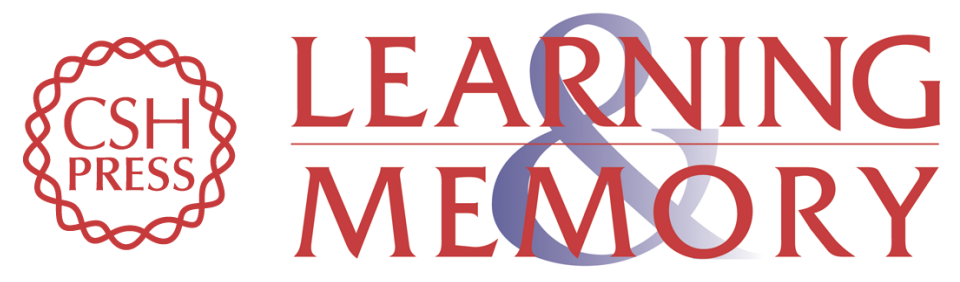

\section{Neural representations of location outside the hippocampus}

James J. Knierim

Learn. Mem. 2006, 13:

Access the most recent version at doi:10.1101/lm.224606

References This article cites 127 articles, 33 of which can be accessed free at: http://learnmem.cshlp.org/content/13/4/405.full.html\#ref-list-1

License

Email Alerting Receive free email alerts when new articles cite this article - sign up in the box at the Service top right corner of the article or click here. 